\title{
Wireless Power Transmission with Short and Long Range Using Inductive Coil
}

\author{
Manish Bhardwaj ${ }^{1}$ Anil Ahlawat ${ }^{2}$ \\ ${ }^{1}$ Computer Science and Engineering, SRM University, Ghaziabad, India \\ ${ }^{2}$ Computer Science and Engineering, KIET, Muradnagar, Ghaziabad, India \\ Email: aapkaapna13@gmail.com, dranilahlawat@gmail.com
}

How to cite this paper: Bhardwaj, M. and Ahlawat, A. (2018) Wireless Power Transmission with Short and Long Range Using Inductive Coil. Wireless Engineering and Technology, 9, 1-9.

https://doi.org/10.4236/wet.2018.91001

Received: December 24, 2017

Accepted: January 22, 2018

Published: January 25, 2018

Copyright (C) 2018 by authors and Scientific Research Publishing Inc. This work is licensed under the Creative Commons Attribution International License (CC BY 4.0).

http://creativecommons.org/licenses/by/4.0/

(c) (i) Open Access

\begin{abstract}
In today's time each individual needs remote framework, yet at the same time control transmission for low power gadgets are wired in nature. Consistent power supply is one of the real issues in the motivation behind the utilization of remote sensor network. Yet, in the power arrangement of remote Sensor Network, the battery has an extremely constrained lifetime and is not supplanted yet by some other persistent power framework. There are separate techniques proposed for shorter and more separate power transmission: Inductive coupling, resonant inductive coupling and air ionization for short separations; microwave and Laser transmission for longer separations. The pioneer of the field, Tesla endeavoured to make a capable, remote electric transmitter more than a century back that has now observed an exponential development. This paper overall lights up all the effective strategies proposed for transmitting power without wires. This study is important for find out the future ways of power transmission. These methods are so important in today's world because of drastic wastage of power. Common wireless power transmission is a point-to-point control transmission. It was demonstrated that the power transmission effectiveness could approach near $100 \%$.
\end{abstract}

\section{Keywords}

WSN, Wireless Power, Non-Radiative, Remote Transmission

\section{Introduction}

Remote power exchange includes the transmission of energy from a power source to an electrical load without connectors, over an air hole. The premise of a remote power framework includes two loops-a transmitter and beneficiary curl. The transmitter curl is stimulated by rotating current to create a magnetic 
field, which thus instigates a current in the beneficiary curl. The ground idea of remote power exchange includes the inductive transmission of vitality from a transmitter to a recipient through a wavering magnetic field. To accomplish this Direct Current (DC), provided by a power source, is changed over into high recurrence Alternating Current (AC) by extraordinarily composed hardware incorporated with the transmitter. The substituting current stimulates a copper wire curl in the transmitter, which creates a magnetic field [1]. As second (recipient) curl is set inside closeness of the magnetic field; the field can incite a substituting current in the accepting curl. The electronic hardware at the receiver side converts that alternate current back to straight current that operates the device. This misfortune is chiefly because of resistive failure and corona failure at the time of transmission of current through wires. When the power transmitted through wires than the current produce heat because of resistance in the wires. Heat produce during the transmission is proportional to multiplication of square of current and resistance with time.

Remote transmission is helpful to control electrical gadgets in situation where interconnecting wires are badly designed, dangerous, or are unrealistic. For instance, the life of Wireless Sensor Network is its node that comprise of a few gadget controllers, memory, sensors, actuators, handsets and battery and battery. The handset can work in four states, i.e. 1) Transmit 2) Receive 3) Idle and 4) Sleep. The real vitality issue of a transmitter of a hub is its getting out of gear state, as in this state it is continually being prepared to get, devouring incredible measure of energy.

To stay away from these misfortunes, researchers are presently a-days attracted towards remote vitality exchange that has a non-resistance condition.

\section{Issues in Wireless Power Transfer}

One of the significant issues arises in the power distribution and the misfortunes occur in this distribution. As the request builds gradually, the power era increments and the power misfortune is likewise expanded. The major measure of energy misfortune happens at the time of transmission and dissemination. The level of loss of energy at the time of transmission and dissemination is approximated as $26 \%$. The productivity of energy transmission can be enhanced to certain level by utilizing high quality composite over head conductors also, underground links that utilization high temperature super conductor. However, the transmission is as yet wasteful. As per World Resources Institution (WRI), India's power matrix has the most elevated transmission and dispersion misfortunes on the planet a whopping $27 \%$. Numbers distributed by different Indian government offices put that number at $30 \%, 40 \%$ and more noteworthy than $40 \%$. This is credited to specialized misfortunes (matrix's wasteful aspects) and burglary. The above talked about issue can be explained by pick an elective alternative for control transmission which could give considerably higher effectiveness, low transmission cost and maintain a strategic distance from control 
robbery. Microwave Power Transmission is one of the promising advancements and might be the exemplary elective for proficient power transmission.

\section{Literature Survey}

J. M. Kim et al. [2] this paper improves the LEACH protocol with the help of cluster head election method. In this paper fuzzy logic technique is used and with the help of this technique, network chooses the cluster head so that lifetime of the network gets increased.

Manish et al. [3] this paper apply a wireless power transfer and Backpressure Technique to reduce the power problem of the network nodes. This paper shows the simulation results by using the energy conservation algorithm. This paper increased the significant lifetime of the network. Link utilization, route maintenance and reduce overhead is the main objective of this paper.

Manish et al. [4] this paper brief the cooperative communication in different nodes which communicate with same data at a single instant of time. This paper uses the optimum relay nodes selection technique in CC network and with the help of this technique, it reduces the power consumption of the network.

Sotiris Nikoletseas et al. [5] this paper focus on wireless charging of the portable devices for this it is work on two different types of protocols one perform wireless charging, evaluate the charging efficiency, and second maintain the balances of the chargers. This paper evaluates the results in real time environment.

Benard Mumo Makaa et al. [6] this paper simply demonstrates the application of solid state tesla coils. According to the results, this paper shows that tesla coils are highly capable of generating high voltage, high-frequency waveforms. It also shows that tesla coils are designed for wireless power transfer.

Xiao Lu et al. [7] this paper discussed the issues related to wireless power charging and development of a commercial product that using this concept of wireless charging. It shows the wireless charging standards and latest network applications which are used in the market. It also discusses the challenges to implementing the wireless charging concept

\section{Tesla's Wireless Power Transfer History}

Tesla spent his assets from development of AC current on his different innovations and finished his endeavors in a noteworthy leap forward in 1899 at Colorado Springs when he propounded to have transmitted 100 million volts of highrecurrence electric power remotely finished a separation of 26 miles at which he had lit up a bank of 200 lights and ran one electric engine. With the Tesla curl, Tesla guaranteed that exclusive $5 \%$ of the transmitted vitality was lost all the while [8]. In any case, broke of assets once more, he searched for speculators to back his task of broadcasting electric power in practically boundless ads up to any point on the globe. The technique he would use to create this remote power was to utilize the world's own particular reverberation with its particular vibra- 
tional recurrence to lead AC power by means of a substantial electric oscillator. Whenever J.P. Morgan consented to endorse Tesla's venture, a bizarre structure was started and practically finished close Wardenclyffe in Long Island, N.Y. Resembling a tremendous cross section like wooden oil derrick with a mushroom top, it had an aggregate stature of 200 feet as shown in Figure 1 and which is called Tesla Tower. At that point abruptly, Morgan pulled back his help to the venture before finishing and Wardenclyffe was closed around 1905. Despite the fact that the venture had not addressed its issues, the beginning of this exploration by Tesla has lit up the researchers and the field has now accomplished more prominent statures with great outcomes.

\section{Classification of Wireless Power Transfer}

All the remote power exchange frameworks require a transmitter to send signals, a recipient to get the signs and a medium. Power can be transmitted over short and also long range.

For short range transmission there are three techniques accessible up until now:

1) Inductive coupling: To transmit control by electromagnetic acceptance.

2) Resonant Inductive Coupling: To transmit control by acceptance between loops at reverberation.

3) Air Ionization: To transmit control by ionizing the medium i.e. air.

For long range transmission there are two strategies proposed:

1) Microwave transmission: To transmit control by long separation control radiating with shorter wavelengths, in microwave run.

2) Laser transmission: To transmit control by changing over power into a laser shaft that is then pointed at a photovoltaic cell.

\subsection{Short Range Transmission}

\subsubsection{Inductive Coupling}

Two conductors are eluded to as common inductively coupled when they are

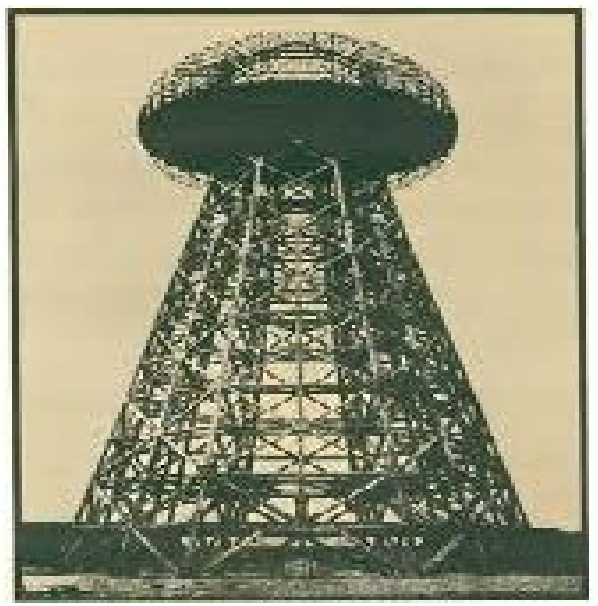

Figure 1. Tesla Tower. 
arranged with the end goal that adjustment in current stream through one wire initiates a voltage over the closures of the other wire through electromagnetic enlistment. In remote exchange, a segment of the attractive transition set up by one circuit interlinks with the second circuit, at that point two circuits are coupled attractively and the vitality is exchanged from one circuit to the another circuit The essentials of this procedure is that the transmitter and recipient curls are inductively coupled. Oscillators are utilized as a part of transmitters to change over DC current to AC current. The AC current go in the transmitter loop produces attractive field, which incites a voltage in beneficiary curl. Magnetic Field is packed in little volume amongst transmitter and collector. The beneficiary has a rectifier those believers Air conditioning once again into DC for utilize. The voltage controller is intended to keep up a steady voltage. The impact of inductance can be amplified or increased through winding the wire. Inductive coupling vitality exchange conveys a far lower danger of electrical stun, at the point when contrasted and conductive charging, on the grounds that there are no uncovered conductors. The principle detriment of this strategy is its lower productivity and expanded resistive warming in contrast with coordinate contact. Inductive charging additionally requires drive hardware and curls that expansion fabricating multifaceted nature and cost. Remote charging cushion, electric brush, transformer work in view of this idea.

\subsubsection{Resonant Inductive Coupling}

Resonant inductive coupling is transmitting power between two loops that are tuned to reverberate at a similar recurrence. Resonance happens when the selfresounding recurrence of loops equivalent to the recurrence of AC control supply, when the equal circuits of loops in high recurrence have the base impedance [9]. At that point, the most vitality will be exchanged from the thunderous way. Full exchange works by influencing a capacitive stacked essential to curl ring with a swaying current as shown in Figure 2.

Resonant Frequency $=$ Capacitance of the plate $*$ Inductance of curl

This produces a wavering attractive field. Since the loop is exceedingly resounding, any vitality set in the curl withers away generally gradually finished a lot of cycles; however in the event that a moment curl is brought close it, the loop can get the majority of the vitality before it is lost, even in the event that it is some separation away. The fields utilized are predominately non-radiative. Attractive resounding coupling can likewise be used to convey control from an expansive source curl to one or numerous little load loops with lumped capacitors at the loop terminals giving a straightforward intends to coordinate full frequencies for the loops. In this strategy, misfortunes happen because of ohmic protection furthermore, radiation. Some of these remote resounding inductive gadgets work at low milliwatt control levels and are battery fueled. Others work at higher kilowatt control levels. All the gadgets which are used at the certain area are get power from the transmitting curl and this transmission is effective upto $75 \%$ as shown in Figure 3. The power transmission between the curl is reduce after a 


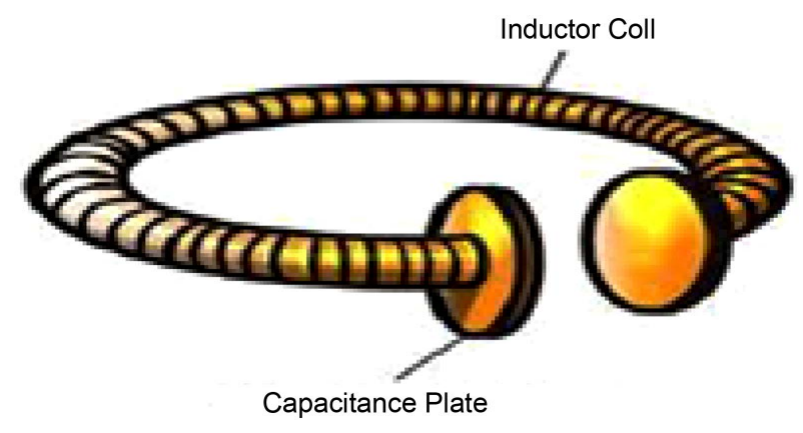

Figure 2. Curl with capacitance plates.

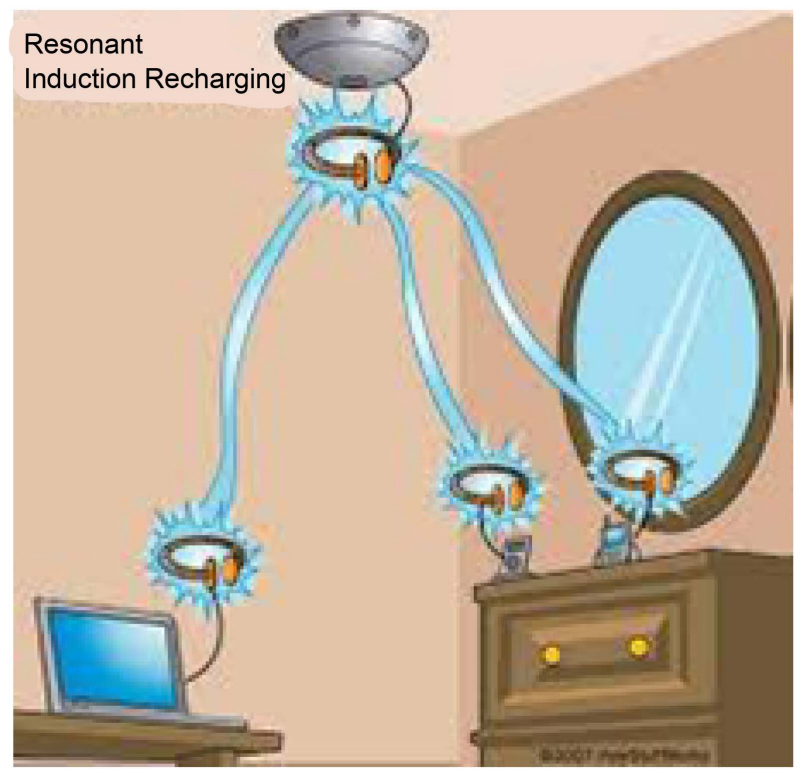

Figure 3. Power transmission with curl.

certain distance that is varies between $10 \mathrm{~cm}$ to $2.2 \mathrm{~m}$.

\subsection{Long Range Transmission}

\subsubsection{Microwave Transmission}

Microwave transmission alludes to the innovation of transmitting data or vitality by the utilization of electromagnetic waves whose wavelengths are advantageously measured in little quantities of centimetre; they are called microwaves. The remote vitality exchange with microwaves requires a wellspring of electromagnetic radiation, and a microwave recipient with a $\mathrm{DC}$ rectifier to change the microwave vitality into DC electrical power [10]. The transmitting and getting units must be in viewable pathway. Viewable pathway (LoS) is a sort of proliferation that can transmit and get information just where transmit and get stations are in perspective of each other with no kind of a hindrance between them. The electrical vitality is first changed over into microwave energy in the transmitter which is transmitted over separation to beneficiary which has rectenna that changes over these microwaves back into electrical vitality. Air conditioning 
can't be changed over specifically to microwave in a transmitter. To start with it must be changed over to DC utilizing oscillator. In the beneficiary, the ouput DC from rectenna is changed over to AC for utilize. Power radiating by microwaves has the trouble that for most space applications the required gap sizes are extensive because of diffraction constraining radio wire directionality. Remote high power transmission utilizing microwaves is well demonstrated. Investigations in the many kilowatts have been performed at Goldstone in California in 1975 and all the more as of late (1997) at Grand Bassin on Reunion Island. These strategies accomplish removes on the request of a kilometre. Under exploratory conditions, microwave transformation proficiency was measured to be around $54 \%$ [11].

\subsubsection{Laser Transmission}

A laser is a gadget that produces light through a procedure of optical enhancement in view of the invigorated outflow of electromagnetic radiation. A laser varies from different wellsprings of light since it radiates light rationally. Spatial rationality enables a laser to be engaged to a tight spot. The instrument of creating radiation in a laser depends on invigorated discharge, where vitality is extricated from a progress in a particle or atom. Power can be transmitted by changing over power into a laser bar that is then pointed at a photovoltaic cell [12]. This component is mostly known as "control radiating" since the power is channeled at a collector that can change over it to electrical energy. There are parcel of favorable circumstances in this framework It permits limit bar traverse huge separations; Compact size; No radiofrequency obstruction to existing radio correspondence [13]. There are different drawbacks too. Laser radiation is unsafe. Change amongst power and light is wasteful. Photovoltaic cells accomplish just $40 \%$-half productivity. Barometrical ingestion, and retention and dissipating by mists, mist, rain, and so on [14]. It requires an immediate viewable pathway with the objective. This technique has been utilized as a part of military and aviation applications.

\section{Advantage and Disadvantage of Wireless Power Transfer}

\subsection{Advantages}

It makes gadgets more helpful and in this manner more alluring to buyers, by killing the requirement for a control line or battery substitution.

- The power disappointment because of short out and blame on links could never exist in transmission.

- Reduction of E-squander by taking out the need of energy ropes [15].

-Wireless charging offers no consumption as the hardware are altogether encased, far from water or oxygen in the environment

\subsection{Disadvantages}

- The capital cost for molecule execution of WPT appears to be high. 
-WPT may cause obstruction with show correspondence frameworks.

- Less effectiveness contrasted with customary charging.

\section{Applications of Wireless Power Transfer}

- Moving targets, for example, fuel free planes, fuel free electric vehicles, moving robots and fuel free rackets.

- Automatic remote charging for portable robots, cordless apparatuses and instrument which wipes out complex components, and work escalated manual energizing and battery substitution [16].

- Another use of WPT are sun based power satellites, vitality to remote regions, communicate vitality all inclusive.

- WPT are utilized for Ubiquitous power source, RF control Versatile Rectifying Circuits (PARC).

\section{Future Scope}

Wireless Power is building a close field remote charging device for purchaser gadgets with the assistance of the Haier gathering, a Chinese hardware maker. Wireless Power illustrated this innovation by remote fuelling a 32-inch TV at a separation of six feet. Delphi Automotives is working with Wireless power to build up a remote charging framework for electric autos. The historic innovation will empower to car maker to coordinate remote dashing into the plan of cross breed and electric vehicles. There is another standard convention for charging cell phone started by the Remote Power Consortium.

\section{Conclusion}

The idea of remote power transmission offers more noteworthy potential outcomes for transmitting power with immaterial misfortunes. In the long run, this could lessen our general public's reliance on batteries, which are at present substantial and costly. As remote innovation is getting mainstream now a day, the request of battery is likewise diminishing. For the long range control transmission power can be sent from source to collectors quickly without wires, lessening the cost. Batteries should be revived or changed in the long run, henceforth the requirement for this sort of work.

\section{References}

[1] Fengyuan, R., Jiao, Z., Tao, H., Chuang, L. and Sajal, K.D. (2011) EBRP: Energy-Balanced Routing Protocol for Data Gathering in Wireless Sensor Networks. IEEE Transactions on Parallel and Distributed Systems, 22.

[2] Chung, M.T., Han, J.Y., Kim, M.J. and Park, H.S. (2008) CHEF: Cluster Head Election Mechansim Using Fuzzy Logic in Wireless Sensor Networks. Proc. of ICACT, 654-659.

[3] Bhardwaj, M. Enhance life Time of Mobile Ad hoc Network Using WiTriCity and Backpressure Technique. Procedia Computer Science, 57, 1342-1350.

[4] Bhardwaj, M. Selection of Efficient Relay for Energy-Efficient Cooperative Ad Hoc 
Networks. American Journal of Networks and Communications. Special Issue: Ad Hoc Networks, 4, 5-11.

[5] Nikoletseas, S. (2015) An Experimental Evaluation of Wireless Power Transfer Protocols in Mobile ad Hoc Networks. IEEE Wireless Power Transfer Conference (WPTC), 1-3.

[6] Makaa, M.B. (2015) Analysis of WPT System Using Rearranged Indirect-Fed Method for Mobile Applications. IEEE Wireless Power Transfer Conference (WPTC), $1-4$.

[7] He, L.M. (2007) Delay-Minimum Energy-Aware Routing Protocol (DERP) for Wireless Sensor Networks. IEEE Eighth ACIS International Conference on Software Engineering, Artificial Intelligence, Networking, and Parallell Distributed Computing. https://doi.org/10.1109/SNPD.2007.526

[8] Manish, B. and Anil, A. (2017) Reduce Energy Consumption in Ad hoc Network with Wireless Power Transfer Concept. International Journal of Control Theory and Applications, 10.

[9] Bhardwaj, M. and Bansal, A. Energy Conservation in Mobile Ad Hoc Network Using Energy Efficient Scheme and Magnetic Resonance. Advances in Networks. Special Issue: Secure Networks and Communications, 3, 34-39.

[10] Bhardwaj, M., Pathak, A. and Saraswat, K.B. (2015) Optimum Experimental Results of AODV, DSDV \& DSR Routing Protocol in Grid Environment. Procedia computer Science, 3rd International Conference on Recent Trends in Computing 2015 (ICRTC-2015), 57, 1342-1350.

[11] Bhardwaj, M. and Singh, A. (2015) Power Management of Ad Hoc Routing Protocols Using Mobility Impact and Magnetic Resonance. Advances in Networks, 3, 27-33.

[12] Han, Z., Kim, I.D., Lu, X., Niyato, D. and Wang, P. (2016) Wireless Charging Technologies: Fundamentals Standards and Network Applications. IEEE Communications Surveys \& Tutorials, 18, 1413-1452. https://doi.org/10.1109/COMST.2015.2499783

[13] Nikoletseas, S., Raptis, P.T. and Raptopoulos, C. (2016) Interactive Wireless Charging for Energy Balance. IEEE 36th International Conference on Distributed Computing Systems (ICDCS), Nara, 27-30 June 2016, 262-270.

[14] Conti, A., Passarella, A., et al. (2009) Power Conservation in Wireless Sensor Networks: A Survey. Ad Hoc Networks, 7, 537-568. https://doi.org/10.1016/j.adhoc.2008.06.003

[15] Ok, C.-S., Lee, S., Mitra, P. and Kumara, S. (2009) Distributed Power Balanced Routing for Wireless Sensor Networks. Computer \& Industrial Engineering, 57, 125-135. https://doi.org/10.1016/j.cie.2009.01.013

[16] Azzi, D., Kanakaris, V. and Ndzi, D. (2010) Ad-Hoc Networks Energy Consumption: A Review of the Ad Hoc Routing Protocols. Journal of Engineering and Technology, 162-167. 